\title{
X-ray spectral and eclipsing model of the clumpy obscurer in active galactic nuclei ${ }^{\star}$
}

\author{
Johannes Buchner ${ }^{1,2}$, Murray Brightman ${ }^{3}$, Kirpal Nandra ${ }^{4}$, Robert Nikutta ${ }^{5,1}$, and Franz E. Bauer ${ }^{1,6,7}$ \\ ${ }^{1}$ Pontificia Universidad Católica de Chile, Instituto de Astrofísica, Casilla 306, Santiago 22, Chile \\ e-mail: johannes . buchner . acad@gmx.com \\ 2 Excellence Cluster Universe, Boltzmannstr. 2, 85748 Garching, Germany \\ 3 Cahill Center for Astrophysics, California Institute of Technology, 1216 East California Boulevard, Pasadena, CA 91125, USA \\ ${ }_{5}^{4}$ Max Planck Institute for Extraterrestrial Physics, Giessenbachstrasse, 85741 Garching, Germany \\ 5 National Optical Astronomy Observatory, 950 N Cherry Avenue, Tucson, AZ 85719, USA \\ 6 Millenium Institute of Astrophysics, Vicuña MacKenna 4860, 7820436 Macul, Santiago, Chile \\ Space Science Institute, 4750 Walnut Street, Suite 205, Boulder, CO 80301, USA
}

Received 3 December 2018 / Accepted 18 July 2019

\begin{abstract}
We present a unification model for a clumpy obscurer in active galactic nuclei (AGN) and investigate the properties of the resulting $\mathrm{X}$-ray spectrum. Our model is constructed to reproduce the column density distribution of the AGN population and cloud eclipse events in terms of their angular sizes and frequency. We developed and released a generalised Monte Carlo X-ray radiative transfer code, XARS, to compute X-ray spectra of obscurer models. The geometry results in strong Compton scattering, causing soft photons to escape also along Compton-thick sight lines. This makes our model spectra very similar to our TORUS previous model. However, only if we introduce an additional Compton-thick reflector near the corona, we achieve good fits to NuSTAR spectra. This additional component in our model can be interpreted as part of the dust-free broad-line region, an inner wall or rim, or a warped disk. It cannot be attributed to a simple disk because the reflector must simultaneously block the line of sight to the corona and reflect its radiation. We release our model as an Xspec table model and present corresponding CLUMPY infrared spectra, paving the way for self-consistent multi-wavelength analyses.
\end{abstract}

Key words. galaxies: active - X-rays: galaxies - methods: numerical

\section{Introduction}

X-ray spectroscopy is a powerful method for characterising the accretion onto supermassive black holes, even when the accretion disk is hidden behind thick columns of gas and dust. For the most heavily obscured Compton-thick active galactic nuclei (CT AGN, $N_{\mathrm{H}} \gtrsim 10^{24} \mathrm{~cm}^{-2}$ ) inference on the accretion and nature of the obscurer requires a correct spectral model for the obscurer (the "torus"). To date, the explored range of X-ray spectral models has been limited to simple geometries, while observations have shown that the real geometry is more complex.

For instance, strong evidence exists today that the obscurer is clumpy and has a large covering factor. The former is found through X-ray eclipse events (e.g. Risaliti et al. 2002; Markowitz et al. 2014), while the latter is evident from the large fractions of obscured AGN found in flux-limited X-ray surveys (e.g. Treister et al. 2004; Brightman et al. 2014; Ueda et al. 2014; Buchner et al. 2015; Aird et al. 2015). Following the orientation-only version of the unification paradigm (strawperson model, Antonucci 1993), a single obscuring structure may explain the diversity of obscured and unobscured AGN as well as eclipse events through sampling different viewing angles. However, such a strict unification across all AGN is ruled out because the accretion luminosity is anti-correlated with the

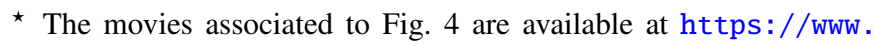
aanda.org and https://doi.org/10.5281/zenodo. 602282 obscured fraction (e.g. Ueda et al. 2003; Hasinger et al. 2005; Buchner et al. 2015). In both the infrared and X-ray regime, many studies have therefore attempted to constrain the covering factor of individual AGN.

Therefore, physically motivated geometric models are needed. Early modelling efforts (see e.g. Matt et al. 2000, and references therein) of the global, low-resolution X-ray spectra at the time employed simplistic models (e.g. slab appropriate for cold reflection from accretion disks; Magdziarz \& Zdziarski 1995). As knowledge and capabilities in spectral and spatial resolution progressed, more realistic modelling was developed, allowing refined parameter constraints and interpretations (e.g. Murphy \& Yaqoob 2009; Brightman \& Nandra 2011a; Ikeda et al. 2009). Studies of nearby (e.g. Matt 2000, and references therein) and distant (e.g. Buchner et al. 2014) obscured AGN show that X-ray spectra are to first order consistent with a photo-electrically absorbed power law, but additionally require varying degrees of Compton-scattering to fit the generally flat spectra and the $\sim 30 \mathrm{keV}$ Compton hump. This can be seen in the literature, for example by many fits preferring the model of Brightman \& Nandra (2011a), BNTORUS, which produces a flat spectrum for Compton-thick obscured AGN, similar to that created by cold disk reflection. Some studies even find additional Compton reflection beyond this model, with examples both in individual local AGN (e.g. Arévalo et al. 2014; Bauer et al. 2015) and large X-ray surveys (e.g. Rivers et al. 2013; Buchner et al. 2014). The arrival of 
NUSTAR (Harrison et al. 2013) revealed, in detail, the complexity of spectral shapes above $10 \mathrm{keV}$ for many local AGN. The analysis of these data is limited by the currently freely available, easy-to-install X-ray spectral models of AGN obscurers. The two physically motivated models most commonly used today are: the BNTORUS model of a bi-conical sphere cutout, and the "donut" geometry of Murphy \& Yaqoob (2009), MYTORUS. Both assume simple geometries and a smooth distribution of obscuring material, which cannot explain AGN with time-variable line-of-sight column densities, commonly interpreted as cloud eclipse events (e.g. Risaliti et al. 2002). A few clumpy geometry models have been recently proposed (e.g. Liu \& Li 2014; Furui et al. 2016), but have not been used to explain eclipse events. To explore various possible geometries, interest has grown in the availability of Monte Carlo codes (e.g. Paltani \& Ricci 2017; Baloković et al. 2018). An extendable open source Monte Carlo simulation package is not available yet.

To compute X-ray spectra we developed a new Monte Carlo simulation code, XARS. We begin in Sect. 2 by describing this code and basic results for inhomogeneous media. In Sect. 3 we construct a clumpy, unified AGN obscurer model which reproduces (1) the column density distribution of AGN at various luminosities and (2) the distribution of cloud eclipse events. Although our initial model spectra produce strong Compton scattering signatures, in Sect. 5 we demonstrate that we must add another reflection component to fit NuSTAR data. We interpret this result and discuss physical origins in Sect. 6.2. Finally, we show how our clumpy obscurer model allows self-consistent infrared \& high-energy studies in combination with CLUMPY models (Nenkova et al. 2008a,b).

\section{Computation of X-ray spectra in inhomogenous media}

\subsection{Simulation code $X A R S$}

We compute the emerging X-ray spectrum for several torus geometries of interest through Monte Carlo simulations. We assume that an isotropically emitting point-source generates photons at the centre of our obscurer geometry. Photoelectric absorption, Compton scattering and line fluorescence (Fe $\mathrm{K} \alpha+\mathrm{K} \beta ; \mathrm{K} \alpha$ of $\mathrm{C}, \mathrm{O}, \mathrm{Ne}, \mathrm{Mg}, \mathrm{Si}, \mathrm{Ar}, \mathrm{Ca}, \mathrm{Cr}$ and $\mathrm{Ni}$ ) are simulated self-consistently as photons pass through matter. The simulation method has already been described in detail in Brightman \& Nandra (2011a), but we provide some computational details in Appendix A. Solar abundances (Anders \& Grevesse 1989) are assumed with cross-sections from Verner et al. (1996).

We make our modular, Python-based simulation code XARS (X-ray Absorption Re-emission Scattering) publicly available ${ }^{1}$. Users can specify their obscurer geometries by implementing a class which prescribes how photons propagate through the medium, and easily change the input abundances and emission lines without modifying the code. Once the Green's functions at each energy grid point and viewing angle have been computed with adequate signal-to-noise, an input photon spectrum, for example a powerlaw, can be applied, and an Xspec grid model compiled.

We verified our code first by comparing with other implementation. We also verified generated spectra from (1) the SPHERE geometry of Brightman \& Nandra (2011a) and Liu \& Li (2015), discussed below, (2) disk reflection off a

\footnotetext{
1 https://github.com/JohannesBuchner/xars
}

semi-infinite plane-parallel slab (Magdziarz \& Zdziarski 1995), discussed in the appendix, (3) reflection off individual spheres (Nandra \& George 1994, see Appendix B) and (4) the biconical cut-out geometry studied in the next section. As a benchmark, for a Compton-thick sphere, XARS can compute very high-quality spectra ( $10^{9}$ photons input across 1000 energy bins) in 90 min on a single $2 \mathrm{GHz}$ laptop $\mathrm{CPU}$, and is trivial to parallelise through independent executions.

\subsection{Inhomogenous geometries leak soft photons}

An interesting test case studied in previous works is a sphere with a biconical cut-out, illustrated in the top left of Fig. 1. The left panel of Fig. 1 compares (3) our results with those of the Geant4-based code of Liu \& Li (2015) (1), grey thick line). For the spectra shown in Fig. 1 we have chosen an edge-on line of sight with column density $N_{\mathrm{H}} N_{\mathrm{H}}=10^{24} \mathrm{~cm}^{-2}$ and a photon index of $\Gamma=1.8$, consistent with Liu \& Li (2015). We find consistent results. In Brightman \& Nandra (2011a), the geometry code erroneously ${ }^{2}$ let photons back-scattered from the inner wall escape without considering re-entry into the torus (2). This affects the geometric interpretability of fits with this model, and potentially the parameters inferred from jointly fit components. Nevertheless, it is very interesting that across the literature this model is found to describe observational data well (e.g., Brightman \& Nandra 2011a,b; Rivers et al. 2013; Buchner et al. 2014; Ricci et al. 2017).

Next we simulated the spectrum of a clumpy model (similar to CLUMPY geometries, see next section for details). We assume spheres of constant density ${ }^{3}$. Our cloud geometry code uses LightRayRider (see Buchner et al. 2017, for details) with parallelised and optimised $\mathrm{C}$ routines to quickly solve the individual photon paths through millions of clouds exactly. However, our tests indicate that beyond $10^{4}$ clouds, the resulting spectrum is not affected any more by the number of clouds. We generated $\sim 10^{9}$ photons for each geometry. For the photons not absorbed inside the obscurer and escaping to infinity we record energy and direction. The LOS column density $N_{\mathrm{H}}$ of that direction from the central source is then computed, and the photon assigned to that particular $N_{\mathrm{H}}$ and altitude angle $\mathrm{bin}^{4}$. In the right panel of Fig. 1 we plot the energy distribution of photons emerging from directions with LOS column densities of $N_{\mathrm{H}}=10^{24} \mathrm{~cm}^{-2}$. Photons originally on paths of lower LOS column densities can be scattered into the LOS. This leads to a substantial component of soft photons proportional to the scattering surface (covering fraction) in (5) clumpy torus models, as noted in previous works (Liu \& Li 2014; Furui et al. 2016). We also consider a smooth torus with gradually varying density layers (4) reproducing the same column density distribution. In contrast to the clumpy model, this geometry blocks soft photons from escaping. In the clumpy model, fluorescent lines are also scattered farther and emerge with strong horns in the spectrum (inset plot of Fig. 1).

\section{Clumpy torus geometry}

\subsection{Cloud population geometry from obscured fractions}

In principle, the construction of a clumpy obscurer model has infinite degrees of freedom, as each location can have an arbitrary density. To simplify this problem, we assume symmetry

\footnotetext{
2 Buchner \& Brightman, priv. comm.

3 See Appendix B for the impact of assuming different density profiles.

4 The models considered here are azimuthally symmetric.
} 

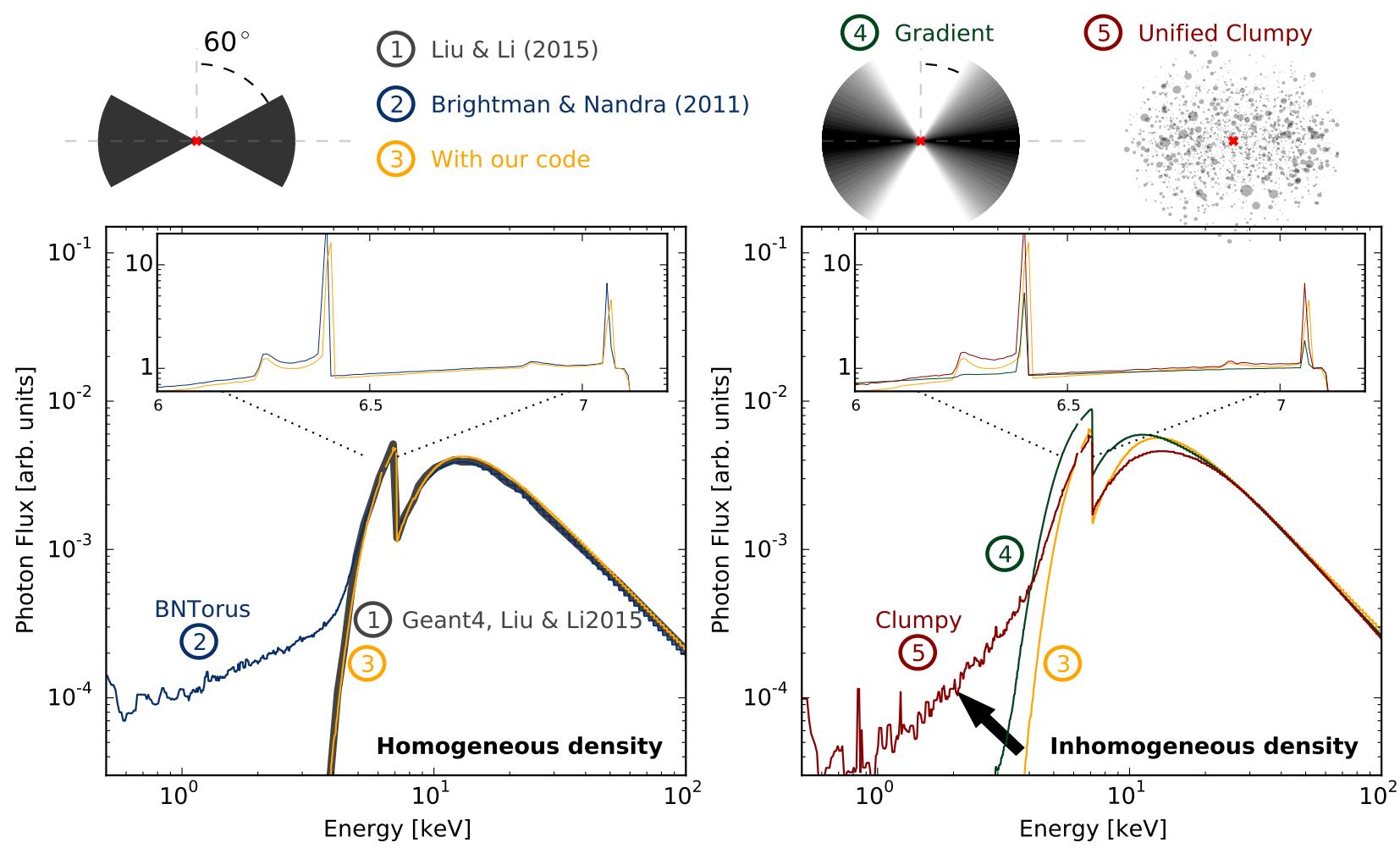

Fig. 1. Simulated X-ray spectra, with geometries shown on top. Left panel: spectra of a Compton-thick bi-cone cut-out geometry in edge-on view. Liu \& Li (2015, shown as (1) demonstrated that the model of Brightman \& Nandra (2011a, shown as (2)) should not leak soft ( $\$ 4 \mathrm{keV})$ photons. We obtained with our code XARS (shown as (3) the same spectrum as Liu \& Li (2015). Right panel: comparison of this uniform density toroid with a gradient toroid (shown as (4)) and our unified clumpy model (shown as (5)). The arrow highlights that, unlike the smooth geometry, leakage of soft photons is possible in the clumpy torus. In the main plots, we removed spectral lines to highlight the continuum shape. The insets show a zoom around the $\mathrm{Fe} \mathrm{K} \alpha$ and $\beta$ features, normalised at $7.07 \mathrm{keV}$. They feature the strongest scattering low-energy tails in the (5) unified clumpy model.

around one axis and that the obscurer column density is, at least on average, a monotonic function with decreasing column density towards the poles. We use the Nenkova et al. (2008b, N08 hereafter) formalism to describe the distribution and properties of clouds. In that formalism, the number of clouds $\mathcal{N}$ seen along a radial line-of-sight is axi-symmetric, and decreases from $\mathcal{N}_{0}$ at the equatorial plane with inclination angle $\beta$ towards the poles, according to a Gaussian function:

$\mathcal{N}=\mathcal{N}_{0} \cdot \exp \left\{-\left(\frac{\beta}{\sigma}\right)^{m}\right\}$

The angular width, $\sigma$, controlling the torus scale height and the average number of clouds in the equatorial plane, $\mathcal{N}_{0}$, are free parameters a priori. We follow previous studies (e.g. N08) which adopted a Gaussian profile $(m=2)$. Using an exponential distribution instead does not affect our conclusions.

To narrow down the possible geometries in a realistic way, we first attempt to fit the model to several known constraints. The first constraint applied is the column density distribution. For luminous (moderately luminous) AGN, we should reproduce the fractions of $25 \%(35 \%)$ for $N_{\mathrm{H}} \gtrsim 10^{24} \mathrm{~cm}^{-2}$ and $30 \%(65 \%)$ at $N_{\mathrm{H}}>10^{22} \mathrm{~cm}^{-2}$ (from Buchner et al. 2015; Aird et al. 2015; Ricci et al. 2015). These are intrinsic fractions (observed, but corrected for selection bias) with the obscuration of the host galaxy subtracted (see Buchner \& Bauer 2017, for details). Simultaneously, the obscurer should allow, albeit rarely, column densities up to $N_{\mathrm{H}}=10^{26} \mathrm{~cm}^{-2}$, since sources with $N_{\mathrm{H}}>10^{25} \mathrm{~cm}^{-2}$ are known (e.g. NGC 1068, Matt et al. 2000). However, when assuming each cloud has the same column density, the column density distribution produced by Eq. (1) is too narrow.

Widening the model column density distribution can be achieved by allowing at a given luminosity a diversity of opening angles or by giving the clouds a diversity in column densities. We choose the latter option, as we prefer to remain with a unification model. Additionally, absorber variability studies show a wide range of preferentially Compton-thin column density changes (e.g. Risaliti et al. 2002, 2005; Markowitz et al. 2014), and Compton-thick variations have also been seen when telescopes with good sensitivity and angular resolution at $>10 \mathrm{keV}$ became available (e.g., Ricci et al. 2016; Marinucci et al. 2016). We therefore allow a range of values for the column density of individual clouds, $N_{\mathrm{H}}^{\text {cloud }}$. Clouds are placed randomly according to the distributions (and re-drawn when overlapping). Fitting the angular width $\sigma$, the total number of clouds and the parameters of a log-normal distribution simultaneously, we find a suitable fit with $10^{5}$ clouds with a 1 dex wide distribution centred at $10^{23} \mathrm{~cm}^{-2}$. This is shown in Fig. 2. Varying the angular width $\sigma$ makes our model (coloured thick curves) reproduce the column density distribution of the bright or faint AGN population (black and grey points, respectively).

The radial cloud distribution was assumed to be uniform across two orders of magnitude. While the model is scale-free, we can define the ratio of outermost-to-innermost radial distances of clouds, $Y=R_{\text {out }} / R_{\text {in }}$. Observations (e.g. Lira et al. 2013; Fuller et al. 2016; García-Burillo et al. 2016; Ichikawa et al. 2016) indicate that the clouds relevant for the infrared radiation extend out up to approximately one order of magnitude farther than the dust sublimation radius, that is, 


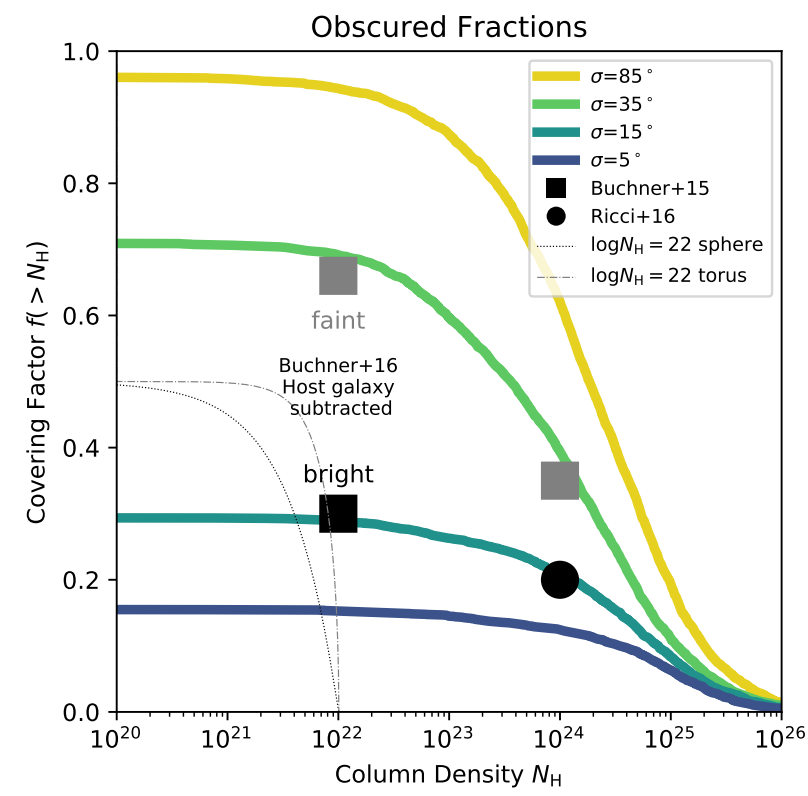

Fig. 2. Column density distribution and associated covering fractions produced by our clumpy model. The curves represent variations of the angular width parameter $\sigma$. Obscured fractions from AGN surveys are shown as black and grey symbols, for bright and faint AGN, respectively. Varying $\sigma$ allows a single instance of our model to reproduce the column density distributions of the population at various luminosities Thin grey curves show the comparatively narrow column density distributions of a single sphere and a torus, each with a maximum crossing column density of $N_{\mathrm{H}}=10^{22} \mathrm{~cm}^{-2}$.

$Y=R_{\text {out }} / R_{\mathrm{d}} \approx 10-20$. For X-rays however, inner ionised clouds 5 can also act as absorbers. We choose $Y=100$ for the X-ray model computation, so that the $\mathrm{X}$-ray obscurer spans two radial orders of magnitude, while the UV/optical, dusty obscurer spans only the last magnitude in radial scale (motivated by Tristram \& Schartmann 2011; Markowitz et al. 2014). For a consistent infrared model, each cloud outside the sublimation radius can be assigned an optical depth of $\tau_{V}$, related to the column density via $\tau_{V}=N_{\mathrm{H}}^{\text {cloud }} /\left(2 \times 10^{21} \mathrm{~cm}^{-2}\right)$, the galactic relationship (Predehl \& Schmitt 1995; Nowak et al. 2012, see also Burtscher et al. 2016).

\subsection{Properties of clouds from eclipse events}

Cloud eclipse events provide constraints on the angular size of individual clouds. In general, the clouds are observed to be very small in angular extent subtended at the black hole $\left(\sim 0.1^{\prime}-1^{\circ}\right.$, Nikutta et al., in prep.). However, observational biases due to limited observing periods preferentially select clouds with high angular velocities and small angular extents. It is furthermore reasonable to assume that the sizes of clouds show a powerlawlike distribution which spans several orders of magnitude. In other words, we expect the observed distribution to only cover the low-end tail of the intrinsic powerlaw angular size distribution. For simplicity, we adopt an exponential distribution of the angular sizes centred around $\theta^{\text {cloud }}=1^{\circ}$, shown in the inset of Fig. 3. This encompasses observed sizes (black histogram). The clouds are assumed to be spherical blobs with diameter $D^{\text {cloud }}=d \cdot \sin \theta^{\text {cloud }}$ for a distance $d$.

Our model is also constructed to reproduce eclipse event rates. The systematic study of cloud events by Markowitz et al.

\footnotetext{
5 e.g. those in the broad-line region (BLR).
}

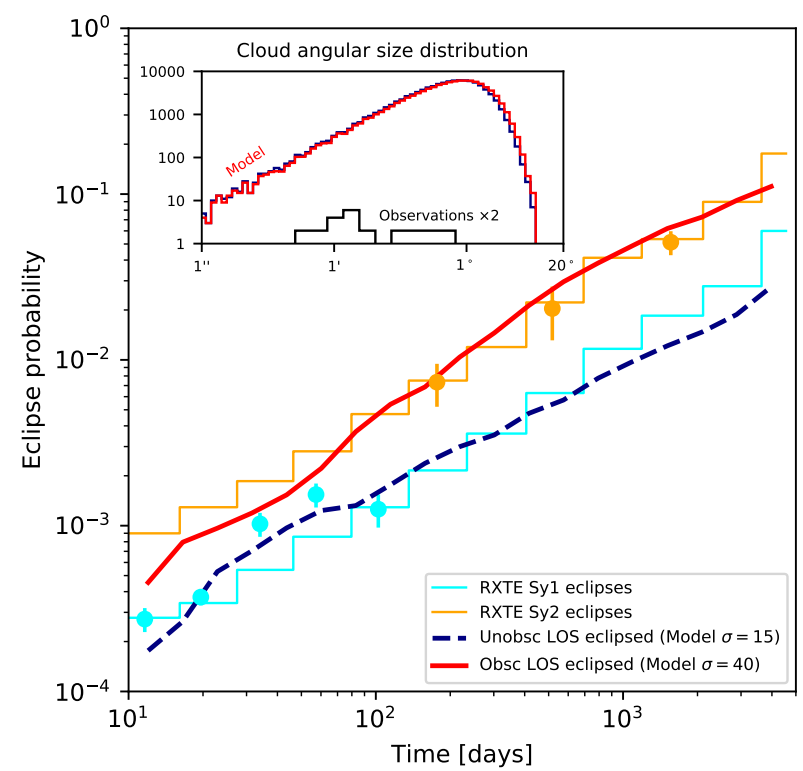

Fig. 3. Predicted rates of eclipses by our model. The probability for an eclipse in a sightline after a given delay is computed, with model clouds on random, circular Keplerian orbits. The systematic study of eclipse event rates measured with the RXTE satellite by Markowitz et al. (2014) are drawn with orange/cyan error bars (type-1/type-2, respectively), with steps approximating their observation window function. We analyse two cases: How frequently an obscured sightline becomes more obscured (red curve) in a high-covering geometry, and how frequently an unobscured sightline becomes obscured in a low-covering geometry (dashed blue curve). The eclipse probability increases with the covering factor. The angular diameter distribution of the model clouds is shown in the inset (blue: low-covering model, red: highcovering model). Sizes of individual observed eclipsing clouds are shown as a black histogram (Nikutta et al., in prep.), covering the lowend tail of the intrinsic distribution.

(2014) found that the probability to see a cloud event increases with the number of days between observations. Figure 3 indicates detected events (error bars) and the sensitivity of their survey based on their windowing function (steps), taken from their Fig. 7. The frequency of events in type 1 (cyan) and type 2 (orange) appear to approximately follow a power law relation. To predict such observables from our clumpy geometry, we assume for simplicity circular Keplerian orbits on random planes of orientation. We set the orbital period of the innermost cloud to one day, which for the outer-most clouds translates to a period of eight years. The corresponding distances ${ }^{6}$ are consistent with observationally inferred distances (approximately light days, see Markowitz et al. 2014, for a review of constraints). We then advance all orbits by a time $\Delta t$ ( $x$-axis) and determine what fraction of 20000 random viewing sight-lines change column density significantly (for type-1: unobscured become obscured, for type-2: obscured at least double the column; obscured refers to the $N_{\mathrm{H}}=10^{22} \mathrm{~cm}^{-2}$ threshold). Because of the different sample selection, we do not assume the same $\sigma$ values as in Fig. 2. Instead, we assume that type- 2 detections are more likely to be created in a more covered torus $\left(\sigma=40^{\circ}\right.$ gives the best fit) than type-1 AGN $\left(\sigma=15^{\circ}\right)$. The slope and eclipse event rates of type-1/type-2 AGN are reproduced, as Fig. 3 demonstrates. When reproducing the data of Markowitz et al. (2014) in Fig. 3, we were free to chose the clumpy model parameter $\sigma$ and the time normalisation. These control the normalisation in the $y$ and

6 The inner most cloud has a orbital radius of $42 \mathrm{au}\left(M_{\mathrm{BH}} / 10^{7} M_{\odot}\right)^{1 / 3}$. 


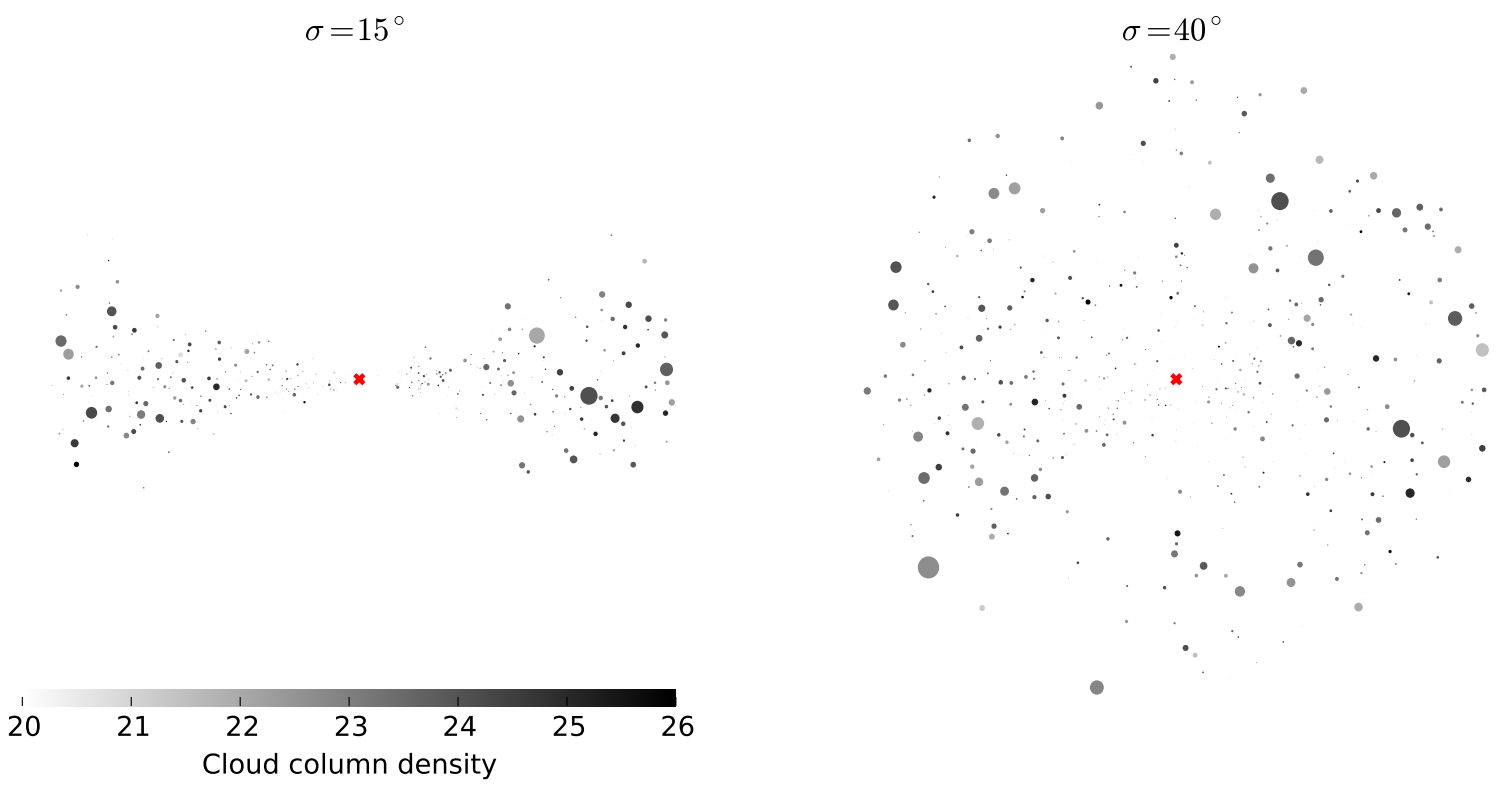

Fig. 4. Cross-sections of clumpy torus model. The left and right geometries differ in the vertical extent $\sigma$, which controls the Gaussian distribution away from the equatorial plane. The red cross indicates the central point source. The clouds vary in size and column density (darkness). A fly-through video is available online.

$x$-axis, respectively. The key result is however that a powerlaw over sufficient orders of magnitudes is predicted, with the correct slope. This result stems from the combination of our assumed exponential angular size distribution and our wide range in distances for the clouds $(Y=100)$ leading to a wide range of orbital and eclipse durations.

A visualisation of our model is presented in Fig. 4. This shows a cross-section through the vertical mid-plane for the two models with angular widths $\sigma=15^{\circ}$ and $40^{\circ}$. Table 2 summarises the assumptions and parameters of our clumpy model.

\subsection{Final model geometry with optional inner ring}

However, the clumpy model constructed above cannot fit some local heavily obscured AGN. As discussed below and in more detail in a companion paper, a highly covering, high-column density $\left(N_{\mathrm{H}} \gtrsim 10^{25} \mathrm{~cm}^{-2}\right)$ reflector is necessary to reproduce the narrow high-energy Compton hump of some local heavily obscured AGN, as traced by $8-10-20 \mathrm{keV}$ X-ray colours (see Buchner et al., in prep.). Briefly speaking, the clumpy structure is composed of a wide range of column densities, together producing a broad Compton hump. See Appendix B for further details regarding the spectrum of reflection off individual clouds, as well as earlier work by Nandra \& George (1994). Introducing an accretion disk that provides reflection to the powerlaw spectrum proved ineffective, because both the intrinsic and reflected emission is strongly attenuated by the torus. Instead, a reflecting mirror is needed that has a virtually unobscured sight-line both to the corona and the observer, while the sight-line between observer and corona is obscured. To address this, we introduce into our clumpy model an optional Compton-thick obscurer. While there are multiple equally satisfactory geometries one could choose for this component, for computational efficiency we compose it also of spheres. As illustrated in Fig. 5, two rings of Comptonthick clouds, just touching each other, form a thick donut. The covering fraction, $C$, determines the radius and number of touching inner clouds used (three to sixteen). This component remains optional so that its presence can be tested by parameter fitting.

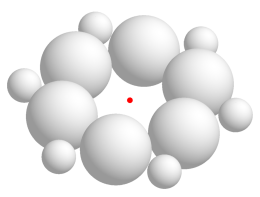

Fig. 5. Compton-thick cloud ring around central point source (red). Here six touching clouds are shown, with a second row of gap-filling clouds. This configuration has a covering factor of $30 \%$. Our model allows variations in the covering factor between $0 \%$ (no cloud ring) and $60 \%$ (3 touching inner clouds).

The discussion in Sect. 6.2 covers physical interpretations of this component.

We compute our final, two-component clumpy model grid across two parameters: (1) the dispersion of the cloud population, TORsigma $\sigma$, and (2) the covering factor of the Comptonthick inner ring, CTKcover $C$, by changing the number of clouds. For computational efficiency, and because the X-ray spectrum remains largely uneffected by this choice, we reduce the number of clouds by a factor of ten to $\mathcal{N}_{\text {tot }}=10^{5}$ and proportionally increase their angular area, setting $\theta^{\text {cloud }}=\sqrt{10} \cdot 1^{\circ} \approx 3^{\circ}$. Column density distributions and cloud eclipse predictions when this component is included are shown in Fig. C.1. To achieve a smoother column density distribution we also altered the cloud $\log$-normal distribution to $N_{\mathrm{H}}^{\text {cloud }}=10^{2} 3.5 \pm 1.0 \mathrm{~cm}^{-2}$.

\section{X-ray spectral model}

\subsection{Model spectrum}

Figure 6 presents various spectra produced by one model geometry $\sigma=30^{\circ}$. We input a powerlaw source of photon index $\Gamma=2$ without a high-energy cut-off. Five LOS column densities are shown, as well as two viewing angles. Towards the Comptonthick regime, a scattering component appears and allows soft photons to escape. Higher column densities also create a deeper trough at $\sim 10 \mathrm{keV}$ relative to the Compton hump. The trough also 


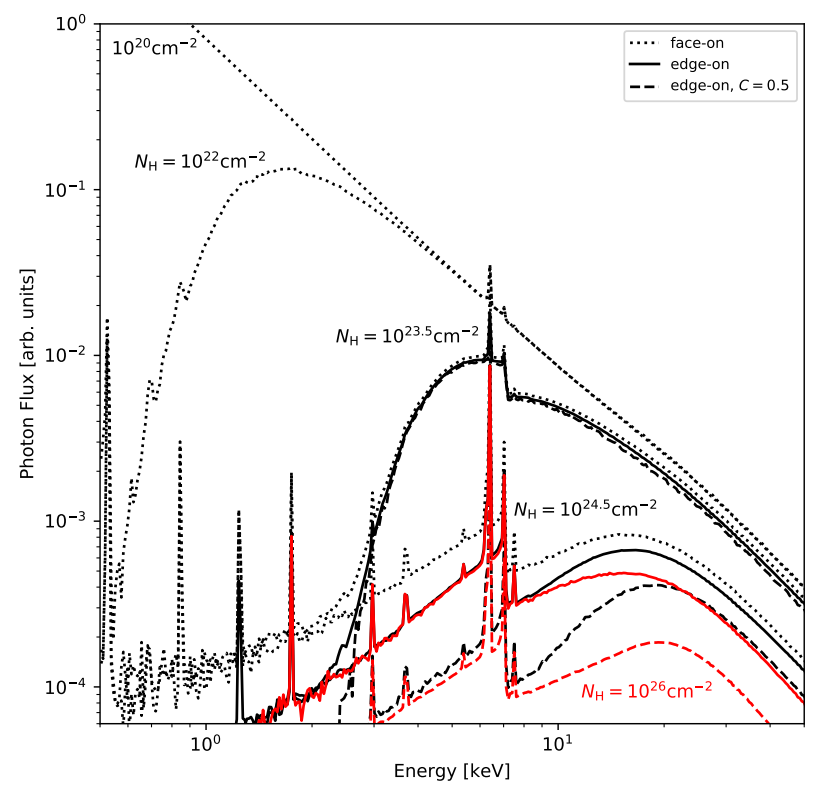

Fig. 6. Clumpy model spectra. Viewing angles (dashed \& solid lines) and LOS column densities (labelled at their respective turn-overs energies) gives diversity in spectral shapes. The highest column density, $N_{\mathrm{H}}=10^{26} \mathrm{~cm}^{-2}$ is shown in red. The lines present typical face-on viewing angles (dotted lines, preferentially low column densities, $0^{\circ}$ ) and edge-on viewing angles (solid and dashed lines, preferentially high column densities, $90^{\circ}$ ). The dashed lines have the inner ring described in Sect. 3.3 enabled.

becomes deeper with $C$, as comparing solid and dashed black curves reveals. In a companion paper, Buchner et al. (in prep.), we explore this trough in detail with a 8-10-20 keV X-ray colourcolour diagram. There we show that local Compton-thick AGN show remarkably flat spectra at $8-10 \mathrm{keV}$, similar to the dashed red curve in Fig. 6, which are difficult to reproduce by existing models. We demonstrate this below in Sect. 5 .

\subsection{X-ray spectral model release}

We release our spectral model, UXCLUMPY (Unified X-ray CLUMPY model), as an Xspec table model for download ${ }^{7}$. The parameters of the model are listed in Table 1.

The model can be used as follows in Xspec syntax:

model atable $\{$ uxclumpy. fits

The model also allows for an exponential high-energy cut-off to more realistically represent the X-ray continuum. This, and the addition of other components, can make model fitting more degenerate and create multiple local optima in parameter space. Therefore, we recommend to initially freeze Ecut to the maximum value. Once a reasonable fit has been obtained, constraining Ecut can be attempted. Unrealistic fits show low photon indices $(\Gamma<1.4)$ and low energy cut-offs to mimic a Compton hump.

Compared to previous spectral models (e.g. BNTORUS, MYTORUS), there are some important differences:

(1) The meaning of $\mathrm{N}_{\mathrm{H}}$ and viewing angle: In our model, the $\mathrm{NH}$ fitting parameter is the LOS column density. A given geometry (TORsigma, CTKcover) produces a diversity of LOS column densities. To understand the implications of the $\mathrm{NH}$ and Theta_inc parameters better, a few technicalities should be mentioned: for each simulated photon escaping to infinity,

7 https://doi.org/10.5281/zenodo. 602282
Table 1. Clumpy model spectral parameters.

\begin{tabular}{cccl}
\hline \hline Name & Symbol & Range & Description \\
\hline PhoIndex & $\Gamma$ & $1-3$ & Photon index \\
Ecut & $E_{\text {cut }}$ & $60-400$ & Energy cut-off $[\mathrm{keV}]$ \\
NH & $N_{\mathrm{H}}(\mathrm{LOS})$ & $10^{20-26}$ & Total LOS column density $\left[\mathrm{cm}^{-2}\right]$ \\
TORsigma & $\sigma$ & $6-90^{\circ}$ & Cloud dispersion \\
CTKcover & $C$ & $0-0.6$ & Covering fraction of inner ring \\
Theta_inc & $\theta_{\text {inc }}$ & $0-90^{\circ}$ & Viewing angle \\
\hline
\end{tabular}

its direction is noted. For the same direction from the X-ray point source, the LOS column density is computed. The photon is then placed in a bin corresponding to column density and viewing angle $\left(0-30^{\circ}\right.$ : face-on, $30-60^{\circ}$ : intermediate, $60^{\circ}-90^{\circ}$ : edge-on). This binning scheme partitions the sky around the corona by column density and viewing angle. In practice, this makes spectral fitting easier, as the LOS column density is the primary influence on spectral shape. Furthermore, physically meaningful fits of column density variations over multiple observation epochs are possible: Unlike existing models, it is not required that the geometry (or equatorial torus column density) changes from source to source, or from observation to observation, or that the viewing angle changes drastically. Instead, multiple LOS column densities are possible at very similar viewing angles, allowing realistic eclipse events by slight rotation self-consistently in our model. We recommend that multi-epoch fits keep the geometry constant, but allow variations in $\mathrm{NH}$.

(2) Opening angles: A key geometric parameter of torus models is the opening angle, $\theta_{\mathrm{op}}$. In our model, the inner Comptonthick covering, CTKcover, can be directly interpreted as a covering factor for that component. The cloud population has however a fuzzy opening angle boundary, which is defined by the angular width $\sigma$ from the plane where the LOS number of clouds decrease to their $1 \sigma$ value. The corresponding opening angle depends on the considered column density and can be read off Fig. 2. Our angular width definition is consistent with the existing CLUMPY model definition (see Sect. 6.1). In the BNTORUS model, the opening angle $\Theta_{\text {tor }}$ can change slightly the Compton hump of that model, and this has been used in some studies to constrain the torus opening angle. In our model, the CTKcover parameter provides a more powerful, geometrically consistent way to probe the reflector geometry. Unfortunately, there exists no simple geometry mapping between the models, as the best-matching photon index is different at the corresponding LOS column densities.

\subsection{Warm mirror component}

Absorbed AGN spectra frequently show excess soft photons. This can be interpreted as a warm mirror: hot electrons Compton scattering the intrinsic powerlaw past the obscurer (e.g. Matt et al. 2000; Bianchi et al. 2006, 2010; Brightman et al. 2014). Frequently this component is simply modelled as a powerlaw with the same photon index as the intrinsic powerlaw but a fraction of the normalisation, following the Thomson scattering approximation. In Xspec, this can be added with:

model atable $\{$ uxclumpy. fits $\}+$ const $*$ zpower

The parameters of the additional powerlaw should be set to the same values as that of the UXCLUMPY obscurer model. 
Table 2. Model parameters.

\begin{tabular}{ccccc}
\hline \hline Description & Distribution & Parameter & Value & Determined from \\
\hline Total number of clouds & Fixed & $\mathcal{N}_{\text {tot }}$ & $10^{5}$ & Column density distribution \\
Vertical distribution & Gaussian & $m$ & 2 & Column density distribution \\
Vertical dispersion & Variable & $\sigma$ & $6^{\circ}-90^{\circ}$ & Column density distribution \\
Radial distribution & Uniform & $q$ & 0 & Assumed \\
Radial extent & Fixed & $Y$ & 100 (X-ray), 10 (IR) & Assumed \\
\hline Cloud sizes & Exponential & $\theta^{\text {cloud }}$ & $1^{\circ}$ & Eclipsing events \\
Column density of clouds & Log-normal & $N_{\mathrm{H}}^{\text {cloud }}$ & $10^{23.5 \pm 1.0}$ & Column density distribution \\
\hline Number of clouds (plane) & (from above) & $\mathcal{N}_{0}$ & $2-9$ & Computed \\
Volume filling factor & (from above) & $f_{V}$ & $2-5 \%$ & Computed \\
Cloud mean optical depth & (from above) & $\tau_{V}$ & 150 & Assuming galactic relation \\
\hline
\end{tabular}

However, if the warm mirror is volume-filling inter-clump hot gas or the narrow-line region, the spectrum is probably more complex, combining warm (hot gas) and cold reflection (dense clouds). As an improved approximation, we sum the emission over all angles $(4 \pi)$ for each geometry. To first order, this angleaveraged spectrum is a powerlaw, because it is dominated by photons escaping without absorption. To second order, a mild Compton hump and Fe $\mathrm{K}$ feature are present, as in the unobscured sight-lines in Fig. 6. To represent the warm mirror more accurately, we provide a second table model that can be added instead of a simple powerlaw:

model atable $\{$ uxclumpy. fits $\}+$ const $*$ at able $\{$ uxclumpy-scattered.fits

All table parameters of the angle-averaged table model (uxclumpy-scattered, warm reflected component) should be linked to their counterparts in the obscurer model (uxclumpy, which includes transmitted and cold reflected component). The relative normalisation constant should not exceed $\sim 10 \%$ to avoid ambiguous powerlaw shapes and unrealistic scattering efficiencies. Additional galactic and warm absorption layers can be added on top as usual.

\section{Fitting local Compton-thick AGN}

The nearby Seyfert 2 Circinus Galaxy (hereafter Circinus) is a prototypical Compton-thick AGN, and due to its proximity has X-ray observations of the nucleus with high signal-to-noise ratios and spatial resolution (see Arévalo et al. 2014, for a recent analysis). The NUSTAR spectrum was extracted following standard procedures with the NUSTAR pipeline (see Brightman et al. 2015, for details).

First, we disable the inner ring component $(C=0$, see Sect. 3.3). Figure 7 presents the best $\mathrm{fit}^{8}$ of this simple clumpy model. In the NuSTAR data the Compton hump begins to rise at relatively high energies $(\sim 10 \mathrm{keV})$, with the model producing a shallower Compton hump. This is caused by the diversity of clouds creating Compton reflection: In low column density clouds, singly and doubly Compton scattered emission can escape. High column density clouds lead to self-absorption and a trough at $8-15 \mathrm{keV}$. In Appendix B we discuss the reflection spectra arising from single clouds in more detail. We find similar results with other Compton-thick AGN (NGC 1068, NGC 424,

\footnotetext{
8 We use the X-ray fitting tools Xspec (Arnaud 1996), and Sherpa (Freeman et al. 2001) with BXA (Buchner et al. 2014), bin spectra to 20 counts, and employ $\chi^{2}$ statistics.
}

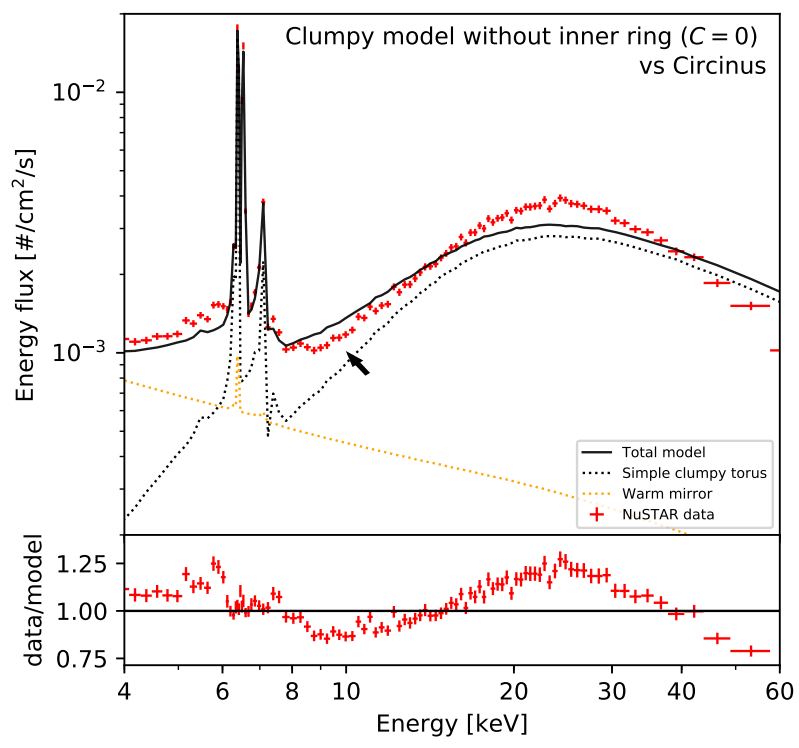

Fig. 7. NUSTAR observations of Circinus fitted with clumpy torus model without inner ring component. For visualisation, the data are binned here to 1000 counts and deconvolved with the model. Additionally, a warm mirror component (orange dotted line, see Sect. 4.3) and lines at rest-frame energy 6.5 and $7.1 \mathrm{keV}$ were added to the fit. The best fit has a LOS column density of $N_{\mathrm{H}}=3 \times 10^{24} \mathrm{~cm}^{-2}$ and a photon index of $\Gamma=1.69$. This model rises already at $\sim 8 \mathrm{keV}$ and produces a shallow Compton hump. In contrast, the data remain flat until $10 \mathrm{keV}$ before rising steeply. This leads to substantial systematic residuals (positive at $6 \mathrm{keV}$, negative at $10 \mathrm{keV}$, positive at $20-30 \mathrm{keV}$ ) in the lower panel.

NGC 3393), and discuss this problem in more detail in a companion paper, using a X-ray colour-colour diagnostic to characterise Compton hump shapes. The spectral shape mismatch results in a poor fit $\left(\chi^{2} /\right.$ d.o.f. $\left.=2774 / 1703 \approx 1.6\right)$. A "pure" high-column density reflector dominating over all contamination by lower column density reflectors (the majority of our current cloud population) is needed. We anticipated the need for such a component in Sect. 3.3. To reduce strong line residuals distracting from the main point of Fig. 7, we included additional gaussian lines. While our model already includes line emission, extended line emission from beyond the torus ( $\gg 10 \mathrm{pc}$ ) is known in this source (Arévalo et al. 2014). Our results and conclusions are consistent with and without these additional lines.

We now refit the data, with the inner ring component enabled. The fit is much better $\left(\chi^{2} /\right.$ d.o.f. $=1765 / 1714 \approx 1.0$, top left panel in Fig. 8), with the residuals (lower panel) not showing systematic deviations. The best-fit has a photon index 

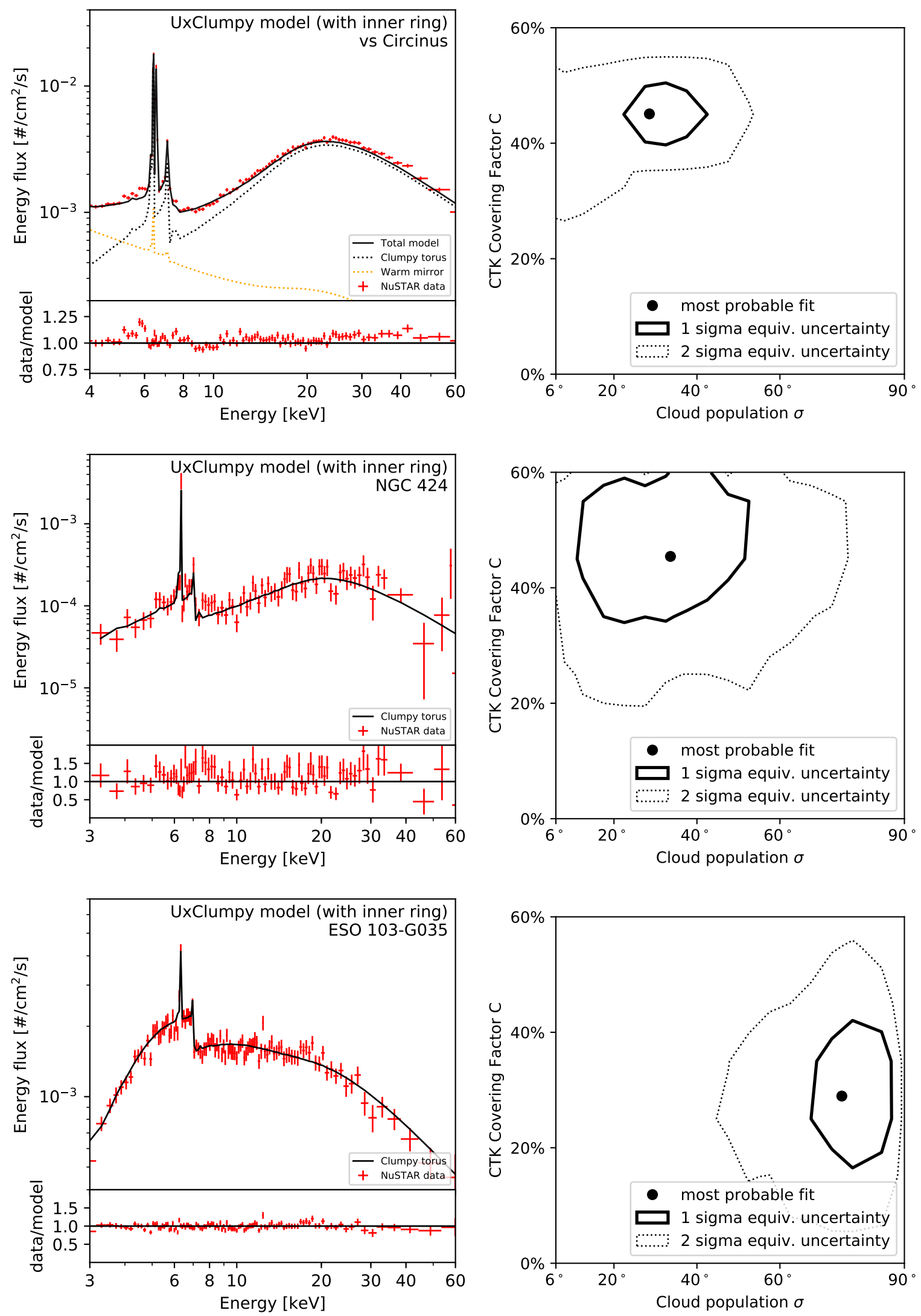

Fig. 8. Left panels: clumpy torus model fit of Circinus, NGC 424 and ESO 103-G035 NuSTAR observations, respectively. In each case a reasonable fit (black line) is obtained with our unified clumpy torus model including an inner ring (see Sect. 3.3). We added a Thomson scattered component (see Sect. 4 for details), which contributes substantially in Circinus. Right panels: constraints on the geometry parameters of the inner ring (CTK covering factor, $y$-axis) and the cloud population $(\sigma, x$-axis), within the allowed ranges of our model. High Compton-thick covering factors are preferred for all three sources. 
of $\Gamma=2.11$, assuming no energy cut-off, behind a line-ofsight column density of $N_{\mathrm{H}}(\mathrm{LOS})=4 \times 10^{24} \mathrm{~cm}^{-2}$. Because the NUSTAR extraction region exhibits substantial contamination by other X-ray sources (Arévalo et al. 2014), we now focus on fitting only the $\geq 8 \mathrm{keV}$ energy range to derive physical properties of the reflector. In this case, we find a photon index of $\Gamma=1.81 \pm 0.10$, behind a line-of-sight column density of $N_{\mathrm{H}}(\mathrm{LOS})=31_{-16}^{+25} \times 10^{24} \mathrm{~cm}^{-2}$. The high-energy cutoff uncertainties, $E_{\text {cut }}=209 \pm 69$, are degenerate with those of $\Gamma$. Importantly, we are able to constrain the geometry of the obscurer (contours in top right panel of Fig. 8). The clumpy component has a covering angle $\sigma$ below $45^{\circ}$, and the Compton-thick covering factor of the inner ring is $C>40 \%$, that is, the corona is extensively covered by Compton-thick material.

To study the soft energy AGN emission as well, we consider NGC 424. Other nearby Compton-thick AGN (such as Circinus) are often contaminated by nearby sources in the host galaxy, such as supernova remnants, ultraluminous $\mathrm{X}$-ray sources and $\mathrm{X}$-ray binaries. NGC 424 is much more luminous than Circinus and therefore its nuclear emission dominates over such host galaxy contaminants. The soft energies show no contamination in XMM-Newton observations down to energies of $\sim 3 \mathrm{keV}$ (Marinucci et al. 2011). The middle left panel of Fig. 8 presents our best fit to NUSTAR spectra in the $3-65 \mathrm{keV}$ range. The fit is of good quality $\left(\chi^{2} /\right.$ d.o.f. $\left.=84 / 79 \approx 1.1\right)$ and gives a photon index of $\Gamma=2.29 \pm 0.14$ without the need for a high-energy cutoff (parameter unconstrained). Such a high photon index is expected from this high-Eddington accretion rate source $\left(L_{\mathrm{bol}} / L_{\mathrm{Edd}} \approx 0.1\right.$, Bian \& Gu 2007; Brightman et al. 2013, 2017). This X-ray emitter is behind a line-of-sight column density of $N_{\mathrm{H}}(\mathrm{LOS})=$ $26_{-20}^{+25} \times 10^{24} \mathrm{~cm}^{-2}$. We are able to constrain the geometry of the obscurer (contours in the centre right panel of Fig. 8): The clumpy component has a covering angle $\sigma<60^{\circ}$, and the Compton-thick covering factor of the inner ring is $C>30 \%$.

We further consider ESO 103-G035, a Compton-thin source. The NUSTAR spectrum of this source is soft without strong obscuration or host contamination, therefore we consider the entire NUSTAR spectrum $(3-70 \mathrm{keV})$. The bottom left panel of Fig. 8 shows our best fit for this source, which is again of good quality $\left(\chi^{2} /\right.$ d.o.f. $\left.=978.58 / 1020 \approx 0.96\right)$ and gives a photon index of $\Gamma=1.84 \pm 0.04$ with a high-energy cutoff $E_{\text {cut }}=209 \pm 69$. This X-ray emitter is behind a line-of-sight column density of $N_{\mathrm{H}}(\mathrm{LOS})=(1.6 \pm 0.1) \times 10^{23} \mathrm{~cm}^{-2}$. We are also able to constrain the geometry of the obscurer in this case (contours in the bottom right panel of Fig. 8): The clumpy component has a covering angle $\sigma>60^{\circ}$ under a face-on viewing angle and the Compton-thick covering factor of the inner ring is $C=28 \pm 7 \%$.

All three spectra prefer large covering fractions of the highcolumn density inner ring reflector. In Compton-thick AGN the cloud population, by means of low $\sigma$ values, tends to be hidden away in the shadow of this inner ring, avoiding low-column density reflection. Indeed, the emerging spectrum and fits change insignificantly when the torus clumps are removed $(\sigma=$ $0)$. In Compton-thin AGN, the cloud population provides the Compton-thin line-of-sight absorber, while the inner ring is again significantly needed as a Compton-thick reflector.

\section{Discussion}

We have presented a clumpy torus model geometry, UXCLUMPY, from which we simulate X-ray spectra. UXCLUMPY provides (1) a wide coverage of energies $(0.1-1000 \mathrm{keV}),(2)$ a wide range of LOS column densities $\left(10^{20-26} \mathrm{~cm}^{-2}\right)$, (3) a variable energy cut-off and (4) fluorescent emission lines self-consistently. Additionally, the clumps in our geometry reproduce the frequency, duration and sizes of eclipse events and X-ray eclipse event observations can be modelled self-consistently without requiring a drastic change in the viewing angle.

The spectral shapes around the Compton hump and trough above the Fe edge differ for the various geometries and viewing angles, and are diverse (see Fig. 6). We additionally compute a better approximation of the warm mirror component. Overall however UXCLUMPY produces spectral shapes similar to the BNTORUS model, which has been found to fit observations well. The added benefit of UXCLUMPY is the ability to infer novel physical characteristics from the spectral fits and consider the energy cut-off. Below we discuss the multi-wavelength spectrum of our model and interpret our inner ring component.

\subsection{Multi-wavelength spectrum}

Infrared and X-ray data can be self-consistently analysed under our clumpy geometry model, by construction. The emerging infrared model was computed using CLUMPY (Nenkova et al. 2008a,b) and is depicted in Fig. 9. CLUMPY performs probabilistic ultraviolet-optical-infrared radiative transfer. This approximation requires that the volume filling factor is small $(<10 \%)$ and the cloud sizes are small, which is the case given our assumed small angular sizes.

Table 2 presents the model parameters and assumptions that went into constructing our clumpy cloud population. For a selfconsistent physical model in the infrared, the CLUMPY torus parameters should be set as follows (see Table 2 ): $Y \approx 10-20$ $(<100), \sigma=$ TORsigma, $N_{0}=2-5$ (depending mildly on $\sigma$ ), $q=0$, and $\tau_{\mathrm{V}} \approx 120-160$.

Figure 9 presents the X-ray model spectrum with the reprocessed infrared emission from the. We normalised at $12 \mu \mathrm{m}$ and use the empirical ratio of 1.7 between the $12 \mu \mathrm{m}$ and intrinsic $2-10 \mathrm{keV}$ luminosity (e.g. Asmus et al. 2015). In the optical and ultraviolet the intrinsic AGN disk emission dominates along unobscured sight-lines (dashed lines in Fig. 9). At long wavelengths, the reprocessed emission is gray-body-like and dominated by the coldest dust regions. In the mid-infrared, selfabsorption in edge-on systems hides the hot inner dust emission, while face-on views expose a range of temperatures. The silicate feature is visible around 10 and $18 \mu \mathrm{m}$. In the X-ray regime very energetic photons can penetrate the obscurer and escape. Between $\sim 0.2-1 \mathrm{~nm}$ a tail of soft photons circumvents the lineof-sight obscurer by being reflected off distant material. In addition to the continuuum, $\mathrm{K}$ shell fluorescence features are clearly visible, the most prominent being Fe K.

We now attempt a multi-wavelength fit of the obscurer from $\mathrm{X}$-rays to infrared. To illustrate the clumpy obscurer, we select NGC 1365, notorious for changing its LOS column density several times over time-scales of days to years (e.g., Risaliti et al. 2005, 2007; Rivers et al. 2015). A successful model should allow the obscurer geometry and inclination to stay the same with only small changes in the sight-line to the corona. Simultaneously, we expect the same geometry to also fit the infrared photometry. Archival photometric data (McAlary et al. 1983; Skrutskie et al. 2006; Ramos Almeida et al. 2009; Asmus et al. 2014, we imposed a $10 \%$ minimum flux uncertainty) and NUSTAR spectra (Rivers et al. 2015, ObsIDs 60002046005,7,9; $5-77 \mathrm{keV}$ ) are presented in Fig. 10. In the three observations (blue, orange, green) in the left of Fig. 10, the spectra differ noticibly in shape. In the insets, the UXCLUMPY parameter probability distributions of a fit to each spectrum are shown 


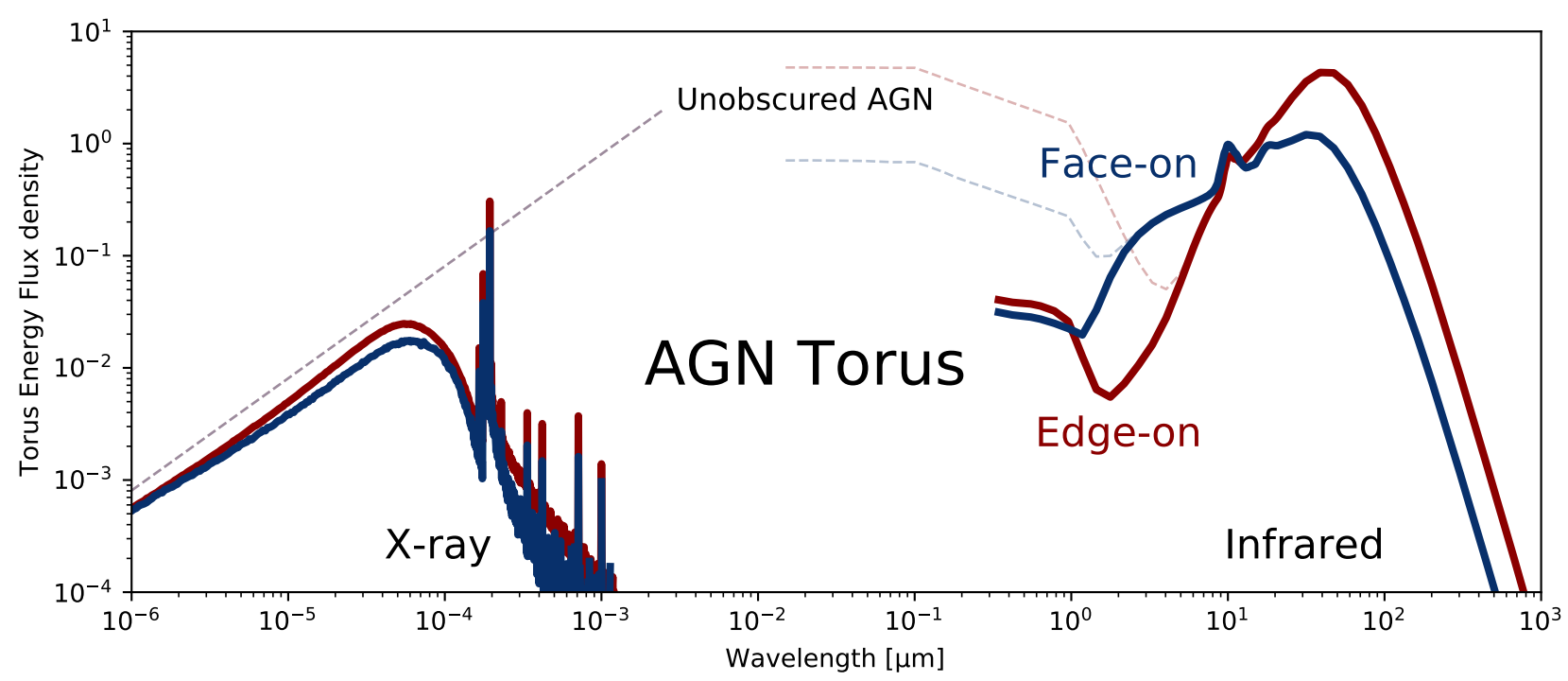

Fig. 9. Multiwavelength spectrum of our unified clumpy geometry. The clumpy torus with $\sigma=30^{\circ}$ (left panel of Fig. 4) is seen through a obscured, Compton-thick line-of-sight either edge-on (red) or face-on (blue). Dashed lines indicate the case of a unobscured view of the accretion disk.

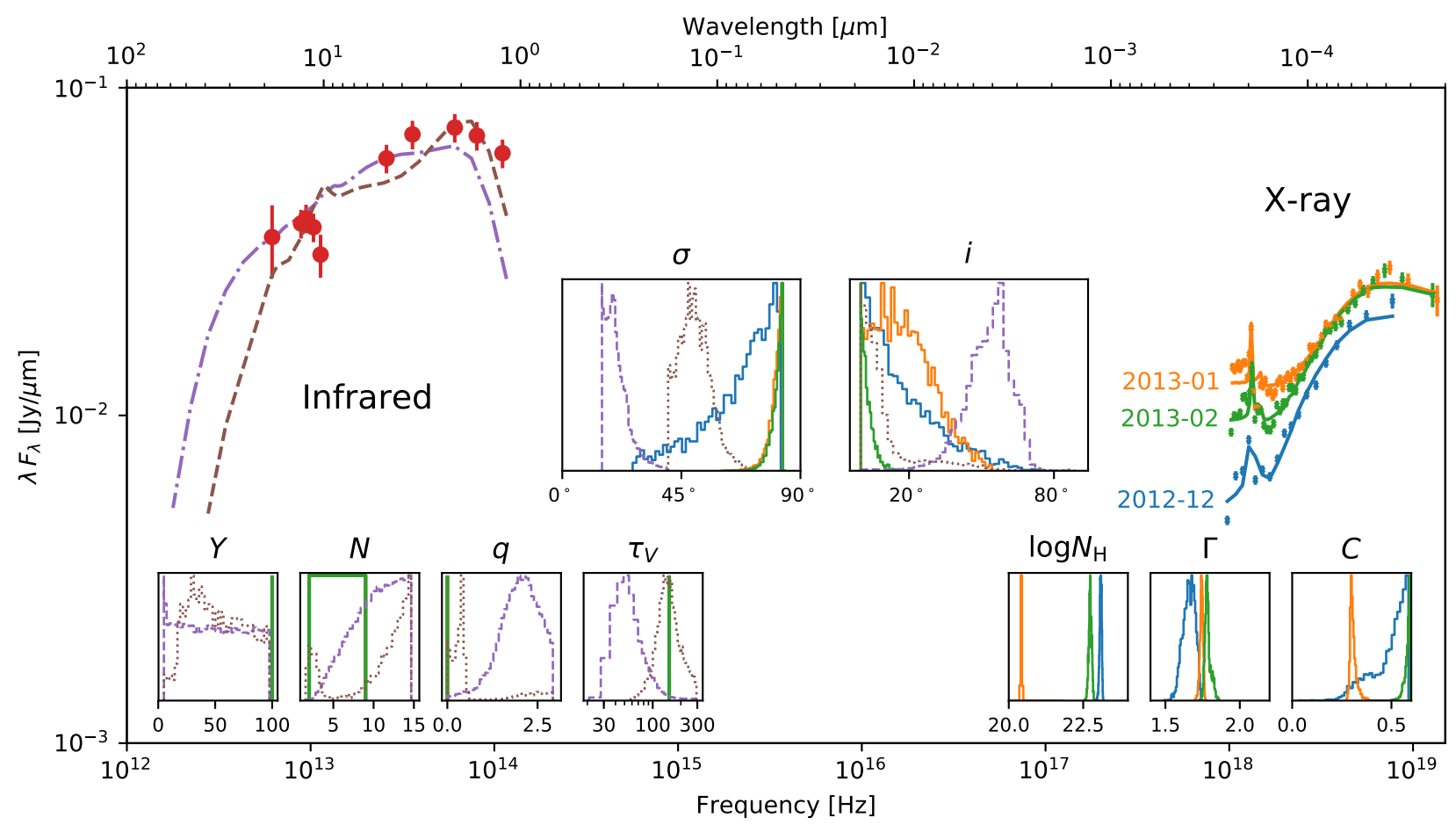

Fig. 10. Multiwavelength fit of our unified clumpy geometry to data of NGC 1365. Infrared photometry on the left overlayed with two CLUMPY fit solutions, and spectra from three NUSTAR observations are shown on the right with UXCLUMPY fits. Insets show parameter probability distributions for each fitted dataset in corresponding colours. The infrared fit solution shown in brown is overall compatible with the X-ray fits (blue, green, orange).

with corresponding colours. Fitting the infrared photometry with CLUMPY leads to two solutions, shown in brown dashed and purple dot-dashed curves. The parameter distributions are again shown in the insets. We compare the solution shown in brown, with a face-on inclination $i<20^{\circ}$, a substantial torus thickness $\sigma>30^{\circ}$ (see central insets of Fig. 10) to the constraints from the X-ray fits (or assumed UXCLUMPY parameters shown in the right insets). Overall, the derived parameters are compatible. This allows the interpretation that a clumpy structure surrounds NGC 1365 whose inner-most clouds vary the X-ray column density and whose outer, dusty clouds irradiate in the infrared. A single geometry, with the same inclination angle was able to simultaneously reproduce column density variations over time and the broad-band X-ray to infrared spectral energy distribution. However, the presented fit does not model all of the complexities present in the data. For example, the least obscured spectrum (orange) shows complex, warm absorption (see Rivers et al. 2015). Additionally, in other variableobscuration sources we find that a multi-wavelength fit gives incompatible geometry constraints, perhaps indicating that the structures differ. This indicates that further modelling is needed and will be addressed in future work. 


\subsection{Interpretation of the inner ring component}

When the inner ring component is present, the X-ray spectrum around the Compton hump is similar to pure cold reflection (e.g. off an infinitely thick slab, or a smooth donut). In the literature such a bulged component has been proposed and interpreted for a variety of reasons. It could be a Compton-thick cloud wind launch site (Krolik \& Begelman 1988), an inner wall (Lightman \& White 1988) or a puffed up inner rim of a hot disk as seen in proto-planetary disks (Dullemond et al. 2001; Dullemond \& Monnier 2010). The spectral shapes are also similar to the spectrum of a warped disk (see Buchner et al., in prep.), and can also be interpreted as one. This is probably the most suitable interpretation for Circinus, where an edge-on maser disk is seen (Greenhill et al. 2003). In the companion paper Buchner et al. (in prep.), a warped disk model provides the best fit.

For compatibility with CLUMPY IR models, we can assume that the inner Compton-thick reflector is dust-free and does not contribute significantly to the infrared spectrum (see simulations of Gallagher et al. 2015, for a component of similar column density). In this case, if it is interpreted as part of a dust-free broad emission line region, very smooth line profiles do not necessitate that it has further discrete clumpy substructure (e.g., Laor et al. 2006). Alternatively, a similar inner ring component was introduced by Pier \& Krolik (1993) to explain the observed, hot $(\sim 1300 \mathrm{~K})$ dust component (see Netzer 2015, for a review); see also Kishimoto et al. (2011) and Hönig et al. (2012) who considered transferring the "puffed-up inner rim" model from protoplanetary disks (Dullemond et al. 2001; Dullemond \& Monnier 2010) to AGN.

Recent interferometric observations in the mid-infrared indicate that the dust emission is in several sources primarily polar with respect to the disk (Asmus et al. 2016; Tristram et al. 2014). Incorporation of these results goes beyond this work. However, clumpy geometries that address these can be calculated rapidly with our XARS code.

\section{Conclusion}

We constructed a clumpy model for the obscurer of AGN which reproduces (1) the column density distribution of the AGN population and (2) observed cloud eclipse events. However, when applying the clumpy model to several nearby, heavily obscured AGN, we find differences between the observed NuSTAR spectra and the model, in particular in the energy region between the Fe K edge and the Compton hump. To resolve this, it is necessary to insert close to the corona a Compton-thick reflector with a substantial covering factor. This new inner component could be interpreted as an inner rim, or a warped or bulged disk. It cannot be attributed to reflection off a simple disk because the reflector must simultaneously block the line of sight to the central engine and reflect it.

The need for the inner Compton-thick reflector (from X-ray spectral fits) and the need for the clumpy component (from eclipses and infrared observations) together could imply three classes of AGN of increasing inclination angle: (1) Unobscured; (2) Obscured by clumps; and (3) Heavily obscured by the inner reflector. The former two can have strong Compton reflection from the inner ring and show eclipses. The latter is reflectiondominated and may produce Compton-thick column density variations (as seen for instance in NGC 1068, Marinucci et al. 2016).

We have developed a powerful Monte Carlo X-ray radiative transfer code, XARS, which can deal with arbitrary geometries. We release this open source software online ${ }^{9}$.

\footnotetext{
9 https://github.com/JohannesBuchner/xars
}

We also publicly release Xspec tables of our UXCLUMPY model online ${ }^{10}$. The spectral model is similar in its ease of use, parameters and spectral shape as the popular BNTORUS model (see Sect. 4). It also allows a physical interpretation of the underlying geometry including covering factors and enables self-consistent analysis of variable column densities (see also Simm et al. 2018). We also note that our geometry has corresponding CLUMPY infrared spectra ${ }^{11}$, paving the way for selfconsistent multi-wavelength analyses.

Acknowledgements. We thank the anonymous referee for careful reading of the manuscript and their insightful comments. JB thanks Marc Schartmann and Leonard Burtscher for discussions on tori in the infrared. JB thanks Eva Lefa for sharing her Monte Carlo code to verify our Compton scattering implementation. JB thanks Mislav Baloković for insightful conversations. We acknowledge support from the CONICYT-Chile grants Basal-CATA PFB-06/2007 \& AFB-170002 (JB, FEB), FONDECYT Regular 1141218 (FEB), FONDECYT Postdoctorados 3160439 (JB) and the Ministry of Economy, Development, and Tourism's Millennium Science Initiative through grant IC120009, awarded to The Millennium Institute of Astrophysics, MAS (JB, FEB). This research was supported by the DFG cluster of excellence "Origin and Structure of the Universe".

\section{References}

Aird, J., Coil, A. L., Georgakakis, A., et al. 2015, MNRAS, 451, 1892 Anders, E., \& Grevesse, N. 1989, Geochim. Cosmochim. Acta, 53, 197 Antonucci, R. 1993, ARA\&A, 31, 473

Arévalo, P., Bauer, F. E., Puccetti, S., et al. 2014, ApJ, 791, 81

Arnaud, K. A. 1996, in Astronomical Data Analysis Software and Systems V, eds. G. H. Jacoby, \& J. Barnes, ASP Conf. Ser., 101, 17

Asmus, D., Hönig, S. F., Gandhi, P., Smette, A., \& Duschl, W. J. 2014, MNRAS, 439, 1648

Asmus, D., Gandhi, P., Hönig, S. F., Smette, A., \& Duschl, W. J. 2015, MNRAS, 454, 766

Asmus, D., Hönig, S. F., \& Gandhi, P. 2016, ApJ, 822, 109

Baloković, M., Brightman, M., Harrison, F. A., et al. 2018, ApJ, 854, A42

Bauer, F. E., Arévalo, P., Walton, D. J., et al. 2015, ApJ, 812, 116

Bian, W., \& Gu, Q. 2007, ApJ, 657, 159

Bianchi, S., Guainazzi, M., \& Chiaberge, M. 2006, A\&A, 448, 499

Bianchi, S., Chiaberge, M., Evans, D. A., et al. 2010, MNRAS, 405, 553

Brightman, M., \& Nandra, K. 2011a, MNRAS, 413, 1206

Brightman, M., \& Nandra, K. 2011b, MNRAS, 414, 3084

Brightman, M., Silverman, J. D., Mainieri, V., et al. 2013, MNRAS, 433, 2485

Brightman, M., Nandra, K., Salvato, M., et al. 2014, MNRAS, 443, 1999

Brightman, M., Baloković, M., Stern, D., et al. 2015, ApJ, 805, 41

Brightman, M., Baloković, M., Ballantyne, D. R., et al. 2017, ApJ, 844, A10

Buchner, J., \& Bauer, F. E. 2017, MNRAS, 465, 4348

Buchner, J., Georgakakis, A., Nandra, K., et al. 2014, A\&A, 564, A125

Buchner, J., Georgakakis, A., Nandra, K., et al. 2015, ApJ, 802, 89

Buchner, J., Schulze, S., \& Bauer, F. E. 2017, MNRAS, 464, 4545

Burtscher, L., Davies, R. I., Graciá-Carpio, J., et al. 2016, A\&A, 586, A28

Dullemond, C. P., \& Monnier, J. D. 2010, ARA\&A, 48, 205

Dullemond, C. P., Dominik, C., \& Natta, A. 2001, ApJ, 560, 957

Freeman, P., Doe, S., \& Siemiginowska, A. 2001, in Society of Photo-optical Instrumentation Engineers (SPIE) Conference Series, eds. J. L. Starck, \& F. D. Murtagh, 4477, 76

Fuller, L., Lopez-Rodriguez, E., Packham, C., et al. 2016, MNRAS, 462, 2618

Furui, S., Fukazawa, Y., Odaka, H., et al. 2016, ApJ, 818, 164

Gallagher, S. C., Everett, J. E., Abado, M. M., \& Keating, S. K. 2015, MNRAS, 451, 2991

García-Burillo, S., Combes, F., Ramos Almeida, C., et al. 2016, ApJ, 823, L12

George, I. M., \& Fabian, A. C. 1991, MNRAS, 249, 352

Greenhill, L. J., Booth, R. S., Ellingsen, S. P., et al. 2003, ApJ, 590, 162

Harrison, F. A., Craig, W. W., Christensen, F. E., et al. 2013, ApJ, 770, 103

Hasinger, G., Miyaji, T., \& Schmidt, M. 2005, A\&A, 441, 417

Hönig, S. F., Kishimoto, M., Antonucci, R., et al. 2012, ApJ, 755, 149

Ichikawa, K., Ricci, C., Ueda, Y., et al. 2016, ApJ, 835, A74

Ikeda, S., Awaki, H., \& Terashima, Y. 2009, ApJ, 692, 608

Kishimoto, M., Hoenig, S. F., Antonucci, R., et al. 2011, A\&A, 536, A78

Krolik, J. H., \& Begelman, M. C. 1988, ApJ, 329, 702

10 https://doi .org/10.5281/zenodo. 602282

11 Available at https://www . clumpy .org 
Laor, A., Barth, A. J., Ho, L. C., \& Filippenko, A. V. 2006, ApJ, 636, 83 Lightman, A. P., \& White, T. R. 1988, ApJ, 335, 57

Lira, P., Videla, L., Wu, Y., et al. 2013, ApJ, 764, 159

Liu, Y., \& Li, X. 2014, ApJ, 787, 52

Liu, Y., \& Li, X. 2015, MNRAS, 448, L53

Magdziarz, P., \& Zdziarski, A. A. 1995, MNRAS, 273, 837

Marinucci, A., Bianchi, S., Matt, G., et al. 2011, A\&A, 526, A36

Marinucci, A., Bianchi, S., Matt, G., et al. 2016, MNRAS, 456, L94

Markowitz, A. G., Krumpe, M., \& Nikutta, R. 2014, MNRAS, 439, 1403

Matt, G. 2000, A\&A, 355, L31

Matt, G., Fabian, A. C., Guainazzi, M., et al. 2000, MNRAS, 318, 173

McAlary, C. W., McLaren, R. A., McGonegal, R. J., \& Maza, J. 1983, ApJS, 52, 341

Murphy, K. D., \& Yaqoob, T. 2009, MNRAS, 397, 1549

Nandra, K., \& George, I. M. 1994, MNRAS, 267, 974

Nandra, K., O’Neill, P. M., George, I. M., \& Reeves, J. N. 2007, MNRAS, 382, 194

Nenkova, M., Sirocky, M. M., Ivezić, Ž., \& Elitzur, M. 2008a, ApJ, 685, 147

Nenkova, M., Sirocky, M. M., Nikutta, R., Ivezić, Ž., \& Elitzur, M. 2008b, ApJ, 685,160

Netzer, H. 2015, ARA\&A, 53, 365

Nowak, M. A., Neilsen, J., Markoff, S. B., et al. 2012, ApJ, 759, 95

Paltani, S., \& Ricci, C. 2017, A\&A, 607, A31

Pier, E. A., \& Krolik, J. H. 1993, ApJ, 418, 673
Predehl, P., \& Schmitt, J. H. M. M. 1995, A\&A, 293, 889

Ramos Almeida, C., Levenson, N. A., Rodríguez Espinosa, J. M., et al. 2009, ApJ, 702, 1127

Ricci, C., Ueda, Y., Koss, M. J., et al. 2015, ApJ, 815, L13

Ricci, C., Bauer, F. E., Arevalo, P., et al. 2016, ApJ, 820, 5

Ricci, C., Trakhtenbrot, B., Koss, M. J., et al. 2017, ApJS, 233, A17

Risaliti, G., Elvis, M., \& Nicastro, F. 2002, ApJ, 571, 234

Risaliti, G., Elvis, M., Fabbiano, G., Baldi, A., \& Zezas, A. 2005, ApJ, 623, L93

Risaliti, G., Elvis, M., Fabbiano, G., et al. 2007, ApJ, 659, L111

Rivers, E., Markowitz, A., \& Rothschild, R. 2013, ApJ, 772, 114

Rivers, E., Risaliti, G., Walton, D. J., et al. 2015, ApJ, 804, 107

Rybicki, G. B., \& Lightman, A. P. 1986, Radiative Processes in Astrophysics (Wiley-VCH)

Simm, T., Buchner, J., Merloni, A., et al. 2018, MNRAS, 480, 4912

Skrutskie, M. F., Cutri, R. M., Stiening, R., et al. 2006, AJ, 131, 1163

Treister, E., Urry, C. M., Chatzichristou, E., et al. 2004, ApJ, 616, 123

Tristram, K. R. W., \& Schartmann, M. 2011, A\&A, 531, A99

Tristram, K. R. W., Burtscher, L., Jaffe, W., et al. 2014, A\&A, 563, A82

Ueda, Y., Akiyama, M., Ohta, K., \& Miyaji, T. 2003, ApJ, 598, 886

Ueda, Y., Akiyama, M., Hasinger, G., Miyaji, T., \& Watson, M. G. 2014, ApJ, 786, 104

Verner, D. A., Ferland, G. J., Korista, K. T., \& Yakovlev, D. G. 1996, ApJ, 465, 487 


\section{Appendix A: Green function computation}

We briefly outline how XARS computes the response spectrum to an input energy as seen from a distant observer. The user provides a function that describes the geometry, and a function that specifies how emerging photons are gridded (e.g. in viewing angle).

Given an input energy, a set of photons is created, typically originating at the geometry origin and directed at all angles uniformly. Then these photons are pumped through the geometry as follows.

The distance traveled by the photon is calculated following an exponential distribution with expectation unity in units of optical depth. The optical depth is defined as the crosssection to both photo-electric absorption and Compton scattering. Because the energies are sufficiently high, electrons appear unbound during scattering, allowing the Klein-Nishina approximation to within a small error (Liu \& Li 2014). With the distance in units of $N_{\mathrm{H}}$ to travel, the geometry user function is called with the current position, direction and distance to travel. It computes the coordinates of the end point. This is achieved by integrating from the start point in the direction until the $N_{\mathrm{H}}$ value has been accumulated. In a simple geometry such as a single unit sphere, the end point is the direction times distance from the current position, where the distance is related to the ratio of $N_{\mathrm{H}}$ to the radial column density through the sphere. In more complex geometries (e.g. many spheres), gaps between objects have to be considered. In any case, the user function returns both the end point and whether the photon has escaped to infinity. After this propagation, all photons still in the medium are interacting with it. Proportional to the relative photo-electric absorption to Compton scattering cross-section at the current energy, the photon is randomly either absorbed or Compton scattered. Compton scattering follows standard formulae (see Rybicki \& Lightman 1986; Brightman \& Nandra 2011a; Paltani \& Ricci 2017) and assigns a new energy and direction. In the case of absorption, there is a chance for the photon to be re-emitted as a fluorescent line. The ratio of the line yield multiplied with abundance to the total absorption cross-section of that element gives the relative probability of line emission (we use the energies and line yields specified in Brightman \& Nandra 2011a). Based on these proportionalities, we distribute the photon to one of the fluorescence lines, with a new randomly drawn direction. In either case (Compton scattering, fluorescent emission), the pumping repeats from the start. If the photon however left the medium, the photon energy, location and direction are passed to the second user function to accumulate the Green function response to the current input energy.

In each pumping step, millions of photons can be processed simultaneously through the use of numpy arrays, making XARS efficient even though it is Python-based.

\section{Appendix B: Irradiation of a single blob under various density profiles}

In this work we have approximated clumps as spheres filled with uniform density profiles. In this section we determine if more realistic profiles without sharp cut-offs (e.g. polytropes) would yield different results. In particular we are interested in the shape of the Compton reflection, and if varying the density profile could alleviate the need for our inner ring component.

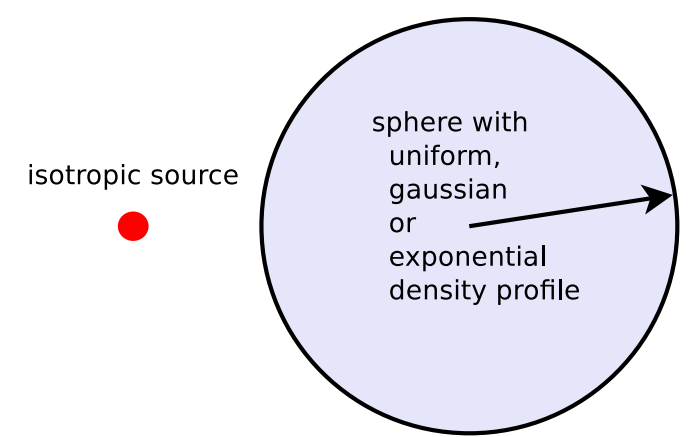

Fig. B.1. Blob irradiation geometry. A isotropic source irradiates the blob from a certain distance, and the spectrum of all reflected photons is assessed in Fig. B.2.

Our test geometry is simple and shown in Fig. B.1: X-rays from an isotropic source irradiate a single, isolated blob. We adopt (1) a uniform density profile, (2) a Gaussian profile and (3) an exponential profile. For the last two we require that the density at the border is $10^{-6}$ of the centre density. The profiles are normalised so that maximum column density is $N_{\mathrm{H}}=$ $10^{23,24,25,26} / \mathrm{cm}^{2}$ (i.e. through the centre). Any reflected/reprocessed X-rays escaping to infinity, from any angle, are collected and their X-ray spectrum determined.

The reflection spectra are shown in Fig. B.2, with the panels corresponding to the three profiles. In each panel we also include reflection off a plane-parallel semi-infinite disk under a $50^{\circ}$ inclination angle (orange line). This is taken from the pexmon spectrum of Magdziarz \& Zdziarski (1995), George \& Fabian (1991), Nandra et al. (2007), and comparable to the angleaveraged spectrum produced by our XARS code of the same geometry (red line). In the top panel, reflection off high-column density uniform blobs (black lines) resembles the disk reflection. At lower column densities (grey) the spectrum peaks at lower energies. The same results were obtained in Nandra \& George (1994). Varying the density profile yields significantly different shapes, as shown in the other panels of Fig. B.2 for Gaussian and exponential profiles. The Compton hump is much weaker, and the spectrum flatter towards low energies. This is not just because these blobs have a lower mass: high-column density results differ throughout also from results of lower column density uniform spheres. The location where reflection primarily occurs is at the surface of uniform blobs, but deeper inside for the other profiles. In the latter, a photon can pass into the blob further, and after scattering may escape off the more common lower column density routes. A single scattering is more likely to put the flight direction of a photon away from the centre than towards it. In the Gaussian/exponential profiles, this decreases the effective column density after each scatter, and subsequently allows high-energy photons to pass through much more easily than in a uniform density profile.

The spectral shape of NUSTAR data studied in this work is similar to the black lines in the left panel, that is, reflection dominated with a strong Compton hump. The addition of the torus ring component emphasises reflection off high-column clouds and surpresses the reflection off low-density clouds (grey in left panel). As Fig. B.2 shows, introducing Gaussian/exponential profile clumps would worsen the situation (as their spectra peak at lower energies) and would under-predict the Compton hump. 

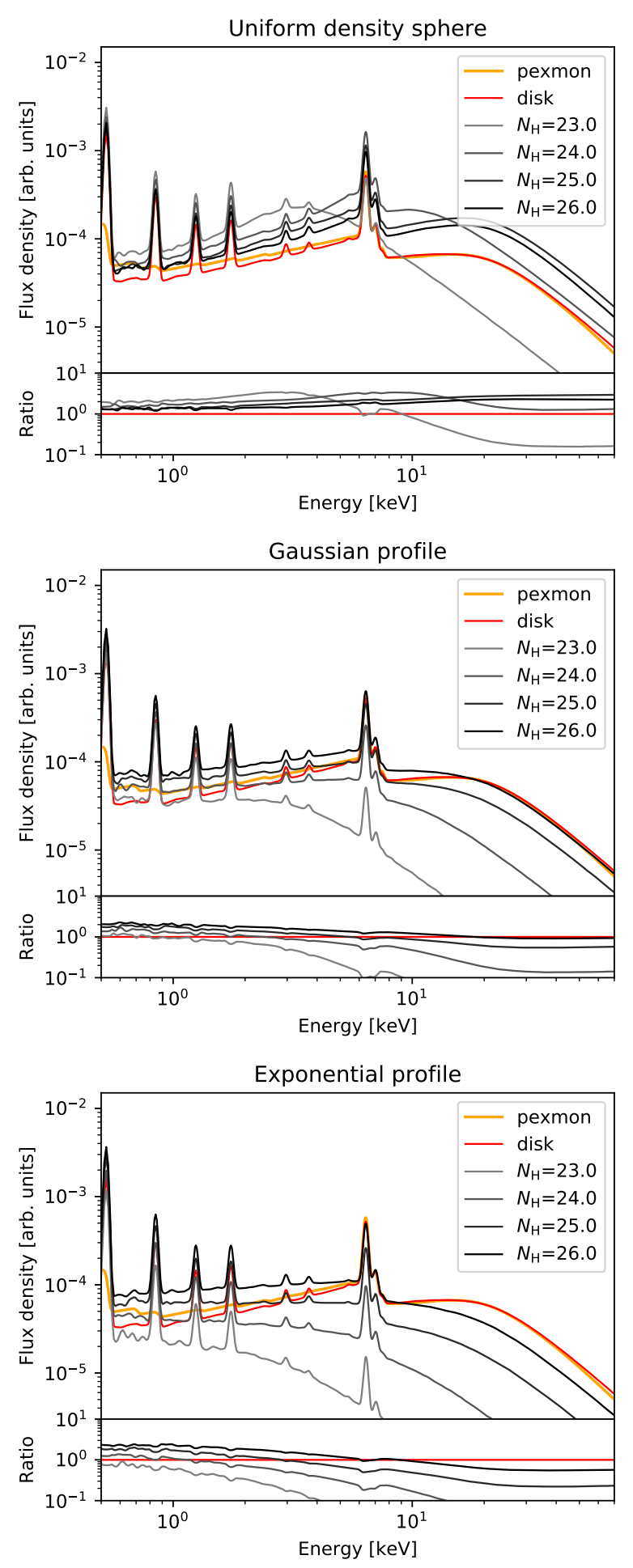

Fig. B.2. Reflection off blobs with various profiles and column densities. For comparison, reflection off a semi-infinite disk is shown in all panels (orange curve: $50^{\circ}$ inclination angle; red curve: angle-averaged, our code).

\section{Appendix C: Clumpy model distributions}

Figure C.1 presents the column density distribution of the final model. Similar to Fig. 2, a wide range of column densities are

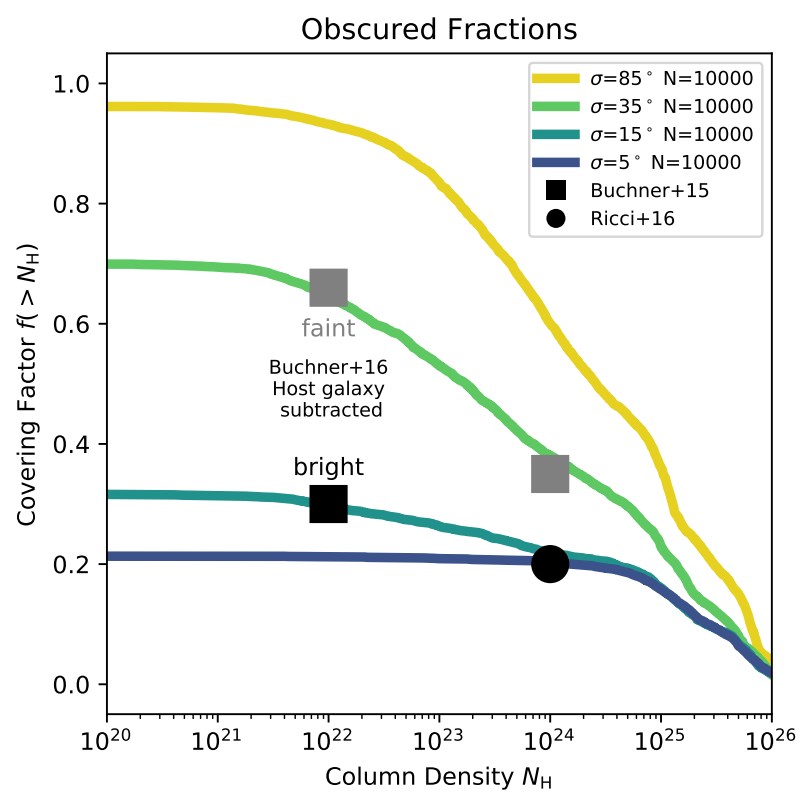

Fig. C.1. Column density distribution and associated covering fractions produced by our clumpy models including the inner ring component. Data points (as in Fig. 2) represent intrinsic AGN obscured fractions at different luminosities. Compared to Fig. 2, the Compton-thick column densities are more frequent because of the inner Compton-thick ring.

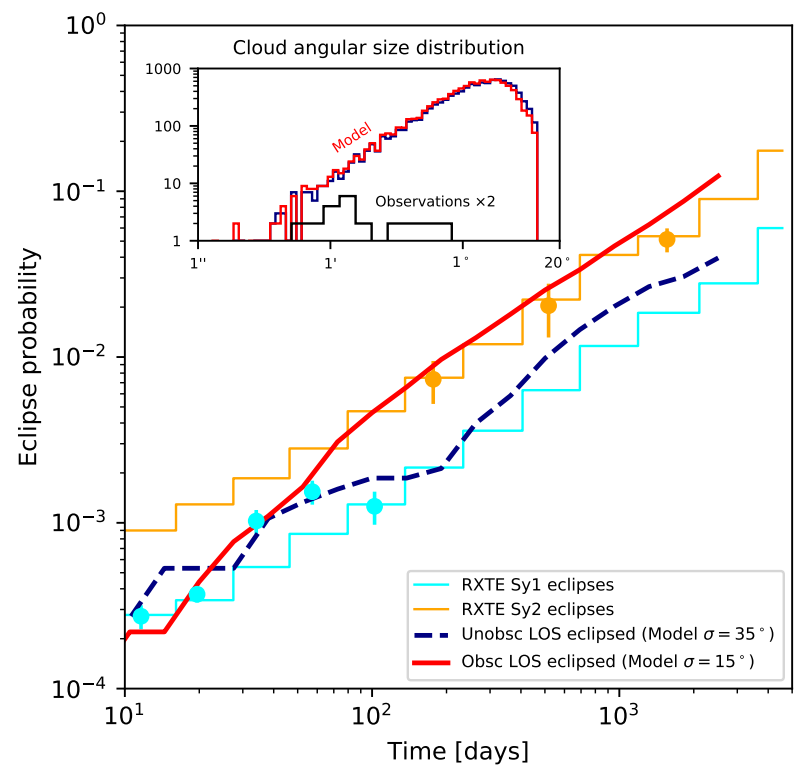

Fig. C.2. Eclipse rates predicted when including the inner ring component. Curves as in Fig. 3, with orange and cyan error bars and curves representing eclipse measurements by Markowitz et al. (2014). Now we show UXCLUMPY with $\sigma=15^{\circ}$ and $\sigma=35^{\circ}$ to reproduce Sy 1 and Sy2, respectively.

produced by each geometry. Now, extremely thick column densities are more common.

Figure C.2 presents the frequency of eclipse of the final model. Similar to Fig. 3, observed cloud sizes and eclipse frequencies can be reproduced, albeit with different model parameters. This is because the inner ring component shades clouds below its covering factor, requiring higher $\sigma$ values. 